In September 2020 the work of the APPG led to a debate in Parliament on delayed diagnosis in axial SpA

Discussions on the future of hydrotherapy services has resulted in the mobilisation of stakeholders across condition areas.

Conclusion: All All Party Parliamentary Group is already proving to be an effective political lever to improve axial SpA care. It has shown huge variations in the standard of care and provision of services still remain and has brought this to the attention of Parliamentarians, policy makers and clinicians.

REFERENCES:

[1] Mohammed H Derakhshan, Himanshu Pathak, Debbie Cook, Sally Dickinson, Stefan Siebert, Karl Gaffney, NASS and BRITSpA investigators, Services for spondyloarthritis: a survey of patients and rheumatologists, Rheumatology, February 2018

[2] Spondyloarthritis in over 16s: diagnosis and management (NG65), https:// www.nice.org.uk/guidance/ng65, February 2017

[3] Spondyloarthritis (QS170), https://www.nice.org.uk/guidance/qs170, June 2018

[4] Axial Spondyloarthritis Services in England - A national inquiry, https:// nass.co.uk/wp-content/uploads/2020/01/Axial-Spondyloarthritis-Services-in-England-FINAL.pdf, January 2020

[5] COVID-19 \& Axial SpA: Government Recommendation Paper, https://nass. co.uk/wp-content/uploads/2020/10/APPG-Recommendation-Paper-COVID-19-Axial-SpA-for-website-1.pdf, October 2020

[6] Helena Marzo Ortega, Simon Whalley, Jill Hamilton, Dale Webb, COVID-19 in axial spondyloarthritis care provision: helping to straighten the long and winding road, https://www.thelancet.com/journals/lanrhe/article/PIIS26659913(20)30413-6/fulltext, 1 December 2020

Disclosure of Interests: Jill Hamilton Grant/research support from: Funding was received from Novartis to support APPG 1, 2 and 4, Dale Webb Grant/research support from: Funding was received from Novartis for APPG 1, 2 and 4, Simon Whalley: None declared

DOI: 10.1136/annrheumdis-2021-eular.2612

\section{OP0277-PARE METABERN, THE EUROPEAN REFERENCE NETWORK FOR RARE HEREDITARY DISEASES: STRUCTURE OBJECTIVES, METABOLIC RMDS AND THE ROLES OF EUROPEAN PATIENT ADVOCACY GROUPS (EPAGS)}

L. Wagner ${ }^{1}$, S. Sestini ${ }^{2}$, C. Brown ${ }^{3}$, A. Finglas ${ }^{4}$, R. Francisco ${ }^{5}$, S. Bond ${ }^{6}$, C. Lampe ${ }^{7}$, C. Belettato ${ }^{6}$, C. Van Lingen ${ }^{6}$, M. Scarpa ${ }^{6}$, on behalf of MetabERN collaboration group. ${ }^{1}$ Deutschsprachige Selbsthilfegruppe für Alkaptonurie e.V., DSAKU e.V., Stuttgart, Germany; ${ }^{2}$ Associazione italiana dei malati di alcaptonuria, AimAKU, Siena, Italy; ${ }^{3}$ Krabbe UK, Krabbe UK, London, United Kingdom: ${ }^{4}$ MSD Action Foundation, MSD Action Foundation, Dublin, Ireland; ${ }^{5}$ Portuguese Association for CDG and Other Rare Metabolic Diseases, APCDG-DMR, Almada, Portugal; ${ }^{6}$ Udine University Hospital, MetabERN, Regional Coordination Center for Rare Diseases, Udine, Italy; ${ }^{7}$ Center for Rare Diseases Giessen, ZSEGI, Giessen, Germany

Background: Inborn metabolic disorders (IMDs) currently encompass more than 1,500 diseases with new ones still to be identified ${ }^{1}$. Each of them is characterised by a genetic defect affecting a metabolic pathway. Only few of them have curative treatments, that target the respective metabolic pathway. Commonly, treatment examples include diet, substrate reduction therapies, enzyme replacement therapies, gene therapy and biologicals, enabling IMD-patient now to survive to adulthood. About $30 \%$ of all IMDs involve the musculoskeletal system and are here referred to as rare metabolic RMDs. Generally, IMDs are very heterogenous with respect to symptoms and severity, often being systemic and affecting more children than adults. Thus, challenges include certified advanced training of adult metabolic experts, standardised transition plans, social support and development of therapies for diseases that do not have any cure yet.

Objectives: Introduction of MetabERN, its structure and objectives, highlighting on the unique features and challenges of metabolic RMDs and describing the involvement of patient representation in MetabERN.

Methods: MetabERN is stratified in 7 subnetworks (SNW) according to the respective metabolic pathways and 9 work packages (WP), including administration, dissemination, guidelines, virtual counselling framework, research/ clinical trials, continuity of care, education and patient involvement. The patient board involves a steering committee and single point of contacts for each subnetwork and work package, respectively ${ }^{2}$. Projects include identifying the need of implementing social science to assess the psycho-socio-economic burden of IMDs, webinars on IMDs and their transition as well as surveys on the impact of COVID- $19^{3}$ on IMD-patients and health care providers (HCPs), social assistance for IMD-patients and analysing the transition landscape within Europe.

Results: The MetabERN structure enables bundling of expertise, capacity building and knowledge transfer for faster diagnosis and better health care. Rare metabolic RMDs are present in all SNWs that require unique treatments according to their metabolic pathways. Implementation of social science to assess the psycho-socio-economic burden of IMDs is still underused. Involvement of patient representatives is essential for a holistic healthcare not only focusing on clinical care, but also on the quality of life for IMD-patients. Surveys identified unmet needs of patient care, patients having little information on national support systems and structural deficits of healthcare systems to ensure HCP can provide adequate clinical care during transition phases. These results are collected by MetabERN and forwarded to the Directorate-General for Health and Food Safety (DG SANTE) of the European Commission (EC) to be addressed further.

Conclusion: MetabERN offers an infrastructure of virtual healthcare for patients with IMDs. Thus, in collaboration with ERN ReCONNET, MetabERN can assist in identifying rare metabolic disorders of RMDs to shorten the odyssey of diagnosis and advise on their respective therapies. On the other hand, MetabERN can benefit from EULAR's longstanding experience regarding issues affecting the quality of life, all RMD patients are facing, such as pain, stiffness, fatigue, rehabilitation, maintaining work and disability claims.

\section{REFERENCES:}

[1] IEMbase - Inborn Errors of Metabolism Knowledgebase http://www.iembase.org/ (accessed Jan 29, 2021).

[2] MetabERN: European Refence Network for Hereditary Metabolic Disorders https://metab.ern-net.eu/ (accessed Jan 29, 2021).

[3] Lampe, C.; Dionisi-Vici, C.; Bellettato, C. M.; Paneghetti, L.; van Lingen, C.; Bond, S.; Brown, C.; Finglas, A.; Francisco, R.; Sestini, S.; Heard, J. M.; Scarpa, M.; MetabERN collaboration group. The Impact of COVID-19 on Rare Metabolic Patients and Healthcare Providers: Results from Two MetabERN Surveys. Orphanet J. Rare Dis. 2020, 15 (1), 341. https://doi. org/10.1186/s13023-020-01619-x.

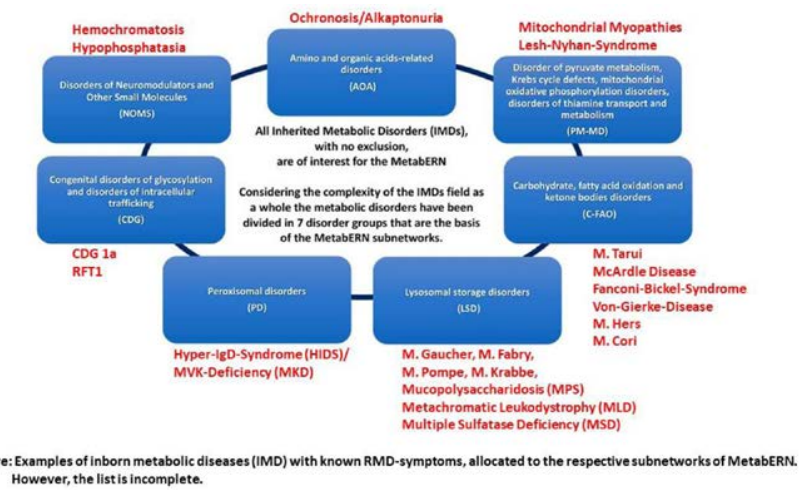

Acknowledgements: The authors thank the MetabERN collaboration group, the single point of contacts (SPOC) of the MetabERN patient board and the Transition Project Working Group (TPWG)

Disclosure of Interests: None declared

DOI: 10.1136/annrheumdis-2021-eular.3372

\section{OP0278-PARE PROVIDING SUPPORT TO FAMILIES OF CHILDREN NEWLY-DIAGNOSED WITH CHILDHOOD ARTHRITIS: A PATIENT AND PARENT-LED PILOT STUDY TO DEVELOP AND ASSESS 'A LITTLE BOX OF HOPE' SUPPORT PACKS}

S. Chibnell-Smith ${ }^{1}$, A. L. Chibnell-Smith ${ }^{1}$, R. Beesley ${ }^{1} .{ }^{1}$ Juvenile Arthritis Research, JAR Project, Tonbridge, United Kingdom

Background: Juvenile Idiopathic Arthritis (JIA) is a heterogenous group of autoimmune disorders characterised by chronic joint inflammation, diagnosed in around 1 in 1,000 children and young people (CYP) under the age of 16. Delays in diagnosis are common [1], awareness is low, and paediatric rheumatologica conditions have a considerable impact on young people and their families [2] A lack of understanding amongst families of newly-diagnosed children leads to uncertainty and anxiety.

Objectives: This patient and parent-led project developed a resource pack for parents of CYP newly-diagnosed with JIA, to provide information and support. Following a pilot, feedback from recipients was collated and analysed to help improve future provision.

Methods: A young person with JIA identified the need for direct family support Juvenile Arthritis Research (a UK charity) developed a unique pack of support information, containing resources for both children and their families - called $A$ Little Box Of Hope. This included information about JIA and support services available for families, as well as Kipo (a children's book about JIA) and accompanying finger puppet. Clinicians at one paediatric rheumatology centre provided information about the packs to newly diagnosed families, who then requested a free box to be posted to them. 